\title{
Successful Use of a GLP-1 Receptor Agonist as Add-on Therapy to Sulfonylurea in the Treatment of KCNJ11 Neonatal Diabetes
}

\author{
Morten Hinds $\varnothing^{1}$, Christoffer Martinussen ${ }^{1}$, Maria Saur Svane ${ }^{1}$, Simon Veedfald ${ }^{2}$, \\ Birthe Gade-Rasmussen ${ }^{1}$, Torben Hansen ${ }^{3}$, Sten Madsbad ${ }^{1}$ \\ ${ }^{1}$ Department of Endocrinology, Hvidovre Hospital, Hvidovre, Denmark \\ ${ }^{2}$ Department of Biomedical Sciences, Faculty of Health and Medical Sciences, University of Copenhagen, Copenhagen, Denmark \\ ${ }^{3}$ The Novo Nordisk Foundation Center for Basic Metabolic Research, Section for Metabolic Genetics, University of Copenhagen, Copenhagen, Denmark
}

Received: $26 / 02 / 2021$

Accepted: 02/03/2021

Published: $31 / 03 / 2021$

How to cite this article: Hindsø M, Martinussen C, Svane MS, Veedfald S, Gade-Rasmussen B, Hansen T, Madsbad S. Successful use of a GLP-1 reception agonist as add-on therapt to sulfonylurea in the treatment of KCNJ11 neonatal diabetes. EJCRIM 2021;8: doi:10.12890/2021_002352.

Conflicts of Interests: The authors did not receive funding for this work. MSS has received funding from the Danish Diabetes Academy for research not related to this work. SM has received research funding from Novo Nordisk and Boehringer-Ingelheim and honoraria from AstraZeneca, MSD, Sanofi and Novo Nordisk for research activities not related to this work.

This article is licensed under a Commons Attribution Non-Commercial 4.0 License

\section{ABSTRACT}

Sulfonylurea monotherapy is the standard treatment for patients with the most common form of permanent neonatal diabetes, KCNJ11 neonatal diabetes, but it is not always sufficient. For the first time, we present a case of successful use of a GLP-1 receptor agonist as add-on therapy in the treatment of a patient with KCNJ11 neonatal diabetes and insufficient effect of sulfonylurea monotherapy. Good glycaemic control was maintained with a HbA1c level of $48 \mathrm{mmol} / \mathrm{mol}(6.5 \%)$ at the end of 26 months' follow-up.

\section{LEARNING POINTS}

- Genetic testing is important in patients with neonatal diabetes.

- Sulfonylurea is the standard treatment for patients with the most common mutation (KCNJ11).

- We present the novel use of a GLP-1 receptor agonist as effective add-on therapy in a patient with KCNJ11 neonatal diabetes and insufficient effect of sulfonylurea monotherapy.

\section{KEYWORDS}

Neonatal diabetes, KCNJ11, sulfonylurea, GLP-1

\section{INTRODUCTION}

Neonatal diabetes occurs in 1 in 125,000 live births ${ }^{[1]}$. Monogenetic defects in the adenosine triphosphate (ATP)-sensitive potassium channel are responsible for $50 \%$ of cases of permanent neonatal diabetes and are most frequently caused by mutations in the potassium inwardly rectifying channel subfamily J member 11 (KCNJ11) gene. KCNJ11 encodes for the Kir6.2-subunit of the pancreatic ATP-sensitive potassium channel, and the mutations impair the ATP sensitivity of the channel and thereby its ability to close ${ }^{[2]}$. This inhibits insulin secretion because the insulin secretion pathway in healthy people is triggered by closure of the ATP-sensitive potassium channel when the intracellular ATP concentration increases as a result of glucose degradation (Fig. 1). Sulfonylureas close the same ATP-sensitive potassium channel by binding to a different subunit (sulfonylurea receptor-1 subunit), and approximately $90 \%$ of patients with KCNJ11 neonatal diabetes can completely switch from insulin to high-dose sulfonylurea ${ }^{[3]}$. However, in a subset of the patients, supplemental insulin is still needed for sufficient 
glycaemic control ${ }^{[3]}$. We present the novel use of a glucagon-like peptide 1 (GLP-1) receptor agonist as add-on therapy to sulfonylurea in a patient with KCNJ11 neonatal diabetes. The case report includes long-term clinical follow-up of the patient as well as evaluation of the effect of sulfonylurea on $\beta$-cell function and postprandial glucose tolerance. The initiation of the GLP-1 receptor agonist treatment in the present case has been briefly reported in Danish ${ }^{[4]}$.

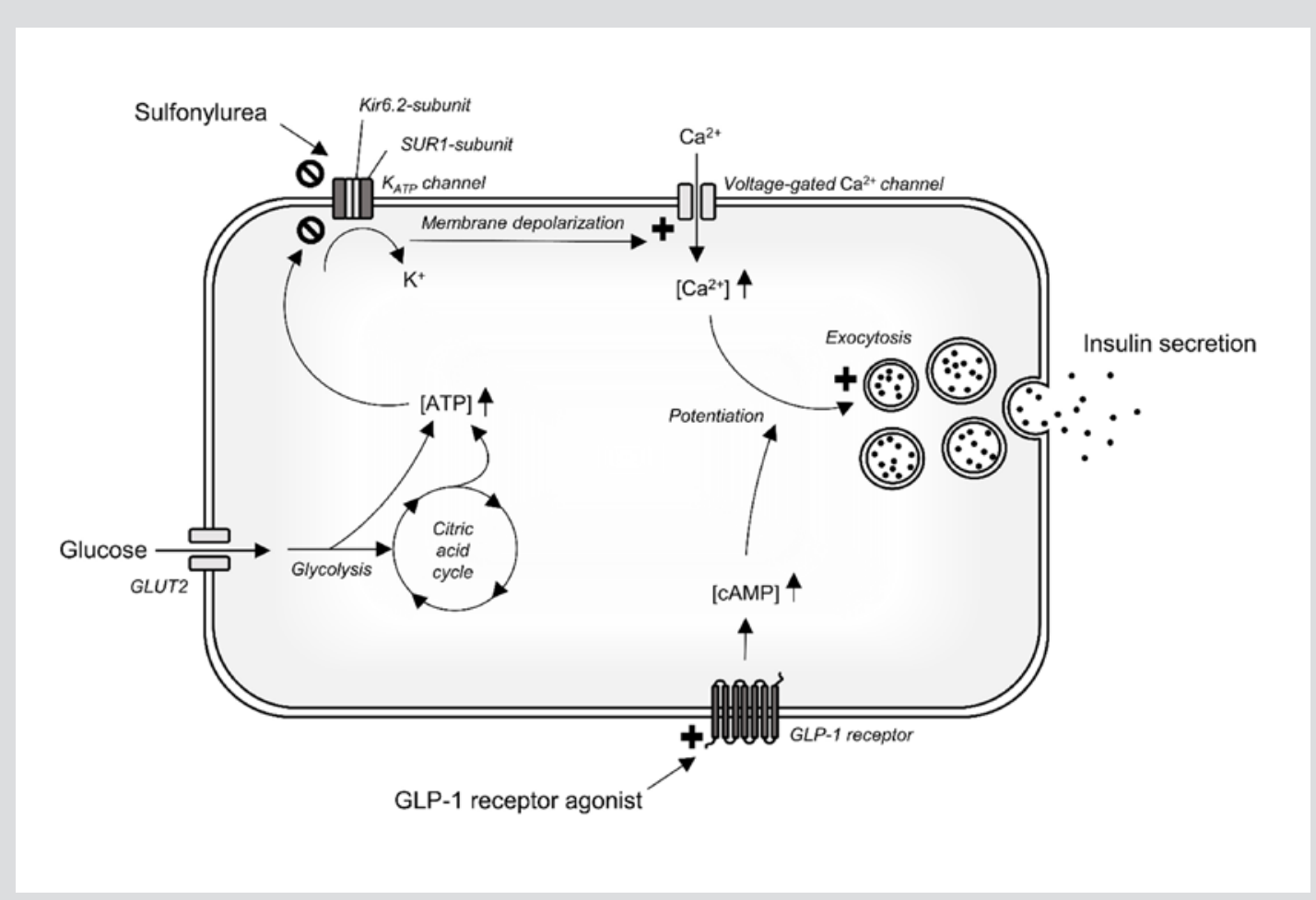

Figure 1. Illustration of selected insulin secretion signalling pathways in the pancreatic $\beta$-cell. Activation of the insulin secretion pathway requires closure of the ATP-sensitive potassium channel which in healthy persons is triggered by glucose degradation leading to increased intracellular ATP. In persons with KCNJ11 mutations, the Kir6.2- subunit of the KATP channel is defective and closure of the channel requires treatment with sulfonylurea, which binds to the SUR1-subunit of the channel. GLP-1 receptor agonists potentate an already active insulin secretion pathway by binding and activating the GLP-1 receptor. ATP, adenosine triphosphate; CAMP, cyclic adenosine monophosphate; GLP-1, glucagon-like peptide 1; GLUT2, glucose transporter 2; KATP, ATP-sensitive potassium channel; SUR-1, sulfonylurea receptor-1

\section{CASE DESCRIPTION}

A 40-year-old lean man (body mass index $22.6 \mathrm{~kg} / \mathrm{m}^{2}$ ) with insulin-treated diabetes mellitus since the neonatal period was seen in the outpatient clinic. He was treated with 60-80 IU insulin daily (38 IU insulin degludec supplemented by prandial bolus insulin aspart), was dysregulated (HbA1c $65 \mathrm{mmol} / \mathrm{mol}(8.1 \%)$ ) and had micro- and macrovascular complications in the form of diabetic retinopathy and a history of ischaemic stroke, respectively.

A test for 65 kDa glutamic acid decarboxylase (GAD65) antibodies was negative, and genetic testing revealed a KCNJ11 R201H mutation. High-dose sulfonylurea treatment was initiated (glibenclamide $59.5 \mathrm{mg}$ daily $(0.8 \mathrm{mg} / \mathrm{kg}$ )) and insulin degludec discontinued. However, $10-$ 12 IU insulin aspart daily was still needed for acceptable glycaemic control. The addition of a dipeptidyl peptidase 4 (DPP4) inhibitor (100 mg sitagliptin) had no effect on glycaemic control and was discontinued. Instead, GLP-1 receptor agonist treatment was initiated (0.3 mg liraglutide). Liraglutide and glibenclamide doses were adjusted, and with the combination of $52.5 \mathrm{mg}$ glibenclamide and $0.6 \mathrm{mg}$ liraglutide, supplemental insulin aspart was no longer necessary. Three months later, $\mathrm{HbA} 1 \mathrm{c}$ was reduced to $48 \mathrm{mmol} / \mathrm{mol}$ (6.5\%) (Fig. 2). GLP-1 receptor agonist treatment was later switched from liraglutide to semaglutide ( $0.5 \mathrm{mg}$ once weekly) to reduce the number of injections. Except for a transient rise in $\mathrm{HbA} 1 \mathrm{c}$ level 6-13 months after the initiation of glibenclamide and GLP-1 treatment, glycaemic control was stable and the $\mathrm{HbA} 1 \mathrm{c}$ level was maintained at $48 \mathrm{mmol} / \mathrm{mol}$ (6.5\%) after 26 months' follow-up. The transient rise in HbA1c was likely influenced by acute appendicitis, 2 weeks with fever and symptoms of viral influenza infection, and an attempt to reduce liraglutide dose in the same time period. The patient was satisfied with the treatment and experienced only mild episodes of hypoglycaemia despite a high level of physical activity. 


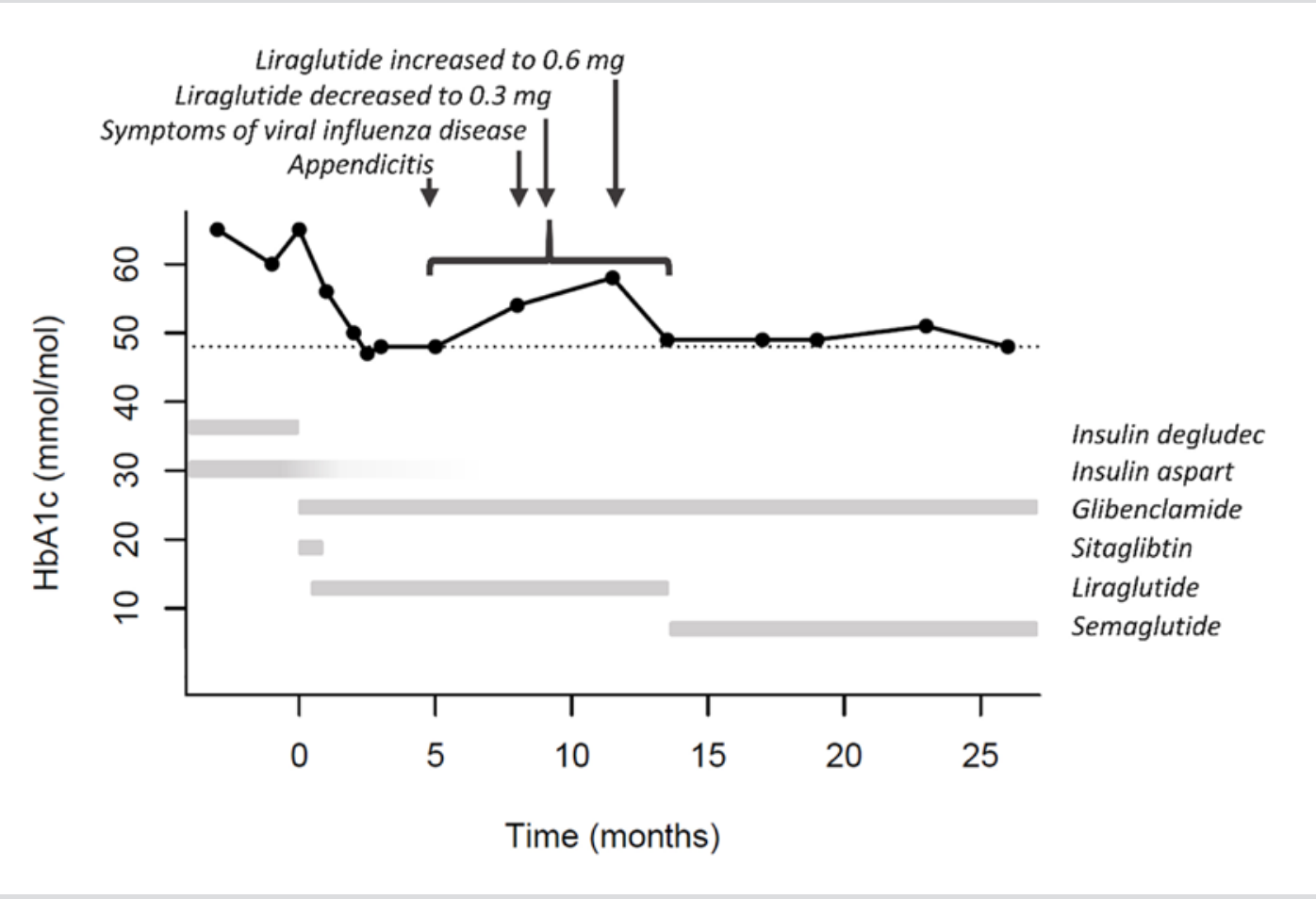

Figure 2. HbA1c response to treatment and intercurrent illness

\section{METHODS AND PROCEDURES}

The effect of glibenclamide on glucose tolerance and $\beta$-cell function was evaluated on separate days by a 3-hour liquid mixed meal test (450 kcal: $49 \mathrm{E} \%$ carbohydrate, $15.8 \mathrm{E} \%$ protein, $35.2 \mathrm{E} \%$ fat) and a $10 \mathrm{~min}$ iv arginine stimulation test ( $5 \mathrm{~g}$ ), respectively. Both tests were performed before (with insulin degludec paused) and 9 months after initiation of glibenclamide treatment (with liraglutide paused and glibenclamide continued). The evening before the liquid mixed meal tests, hyperglycaemia was controlled by the administration of isophane insulin and insulin aspart, whereas no insulin was administered before the arginine stimulation tests. The patient fasted overnight before test days. Blood was sampled for analysis of plasma glucose (YSI model 2300 STAT plus; YSI, Yellow Springs, Ohio, USA, www.ysi.com) and serum C-peptide concentrations (IMMULITE® 2000 analyzer; Siemens Healthcare Diagnostics, Tarrytown, NY, USA, www.siemens-healthineers. com). The total area under the curve (tAUC) was calculated using the trapezoidal rule.

\section{RESULTS}

Glibenclamide treatment reduced plasma glucose (peak: pre 18.3 vs post $10.8 \mathrm{mmol} / \mathrm{l}$, 2-hour: pre $17.3 \mathrm{vs}$ post $10.7 \mathrm{mmol} / \mathrm{l}$, tAUC: pre $2781 \mathrm{vs}$ post $1635 \mathrm{mmol} / \mathrm{l} \cdot \mathrm{min}$ ) and augmented serum C-peptide concentrations (peak: pre 273 vs post $788 \mathrm{pmol} / \mathrm{l}$, tAUC: pre $31.5 \mathrm{vs} \mathrm{post}$ $126 \mathrm{nmol} / \mathrm{l} \cdot \mathrm{min}$ ) during the liquid mixed meal test (Fig. 3A,B). Moreover, glibenclamide improved maximal $\beta$-cell function represented by a six-fold increase in peak C-peptide concentrations during the arginine stimulation test (pre 291 vs post 1761 pmol/l) despite slightly lower plasma glucose concentrations (Fig. 3C,D).

\section{DISCUSSION}

In this case of a 40-year-old patient with dysregulated insulin-treated diabetes and micro- and macrovascular complications, the diagnosis of a KCNJ11 R201H mutation enabled improved glycaemic control through targeted treatment. High-dose sulfonylurea improved, but did not normalize, $\beta$-cell function and glucose tolerance, and supplemental insulin treatment was still required until GLP-1 receptor agonist treatment was initiated. While the use of GLP-1 receptor agonists in the treatment of KCNJ11 neonatal diabetes has never been described, a single case of successful use of the DPP4 inhibitor sitagliptin as add-on therapy to sulfonylurea has been reported ${ }^{[5]}$. This contrasts with our case where sitagliptin did not sufficiently potentiate the effect of glibenclamide. 
A

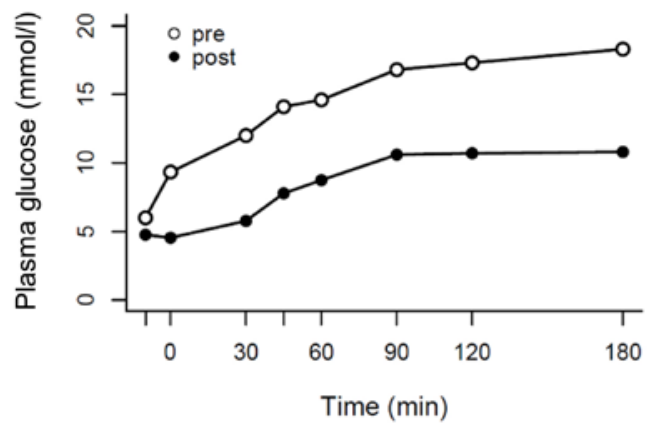

B

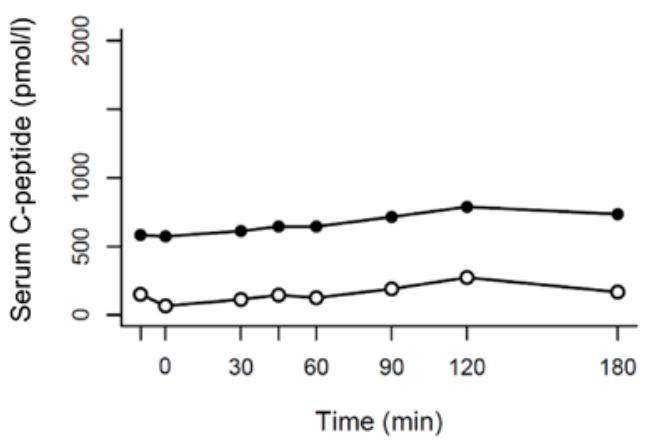

C

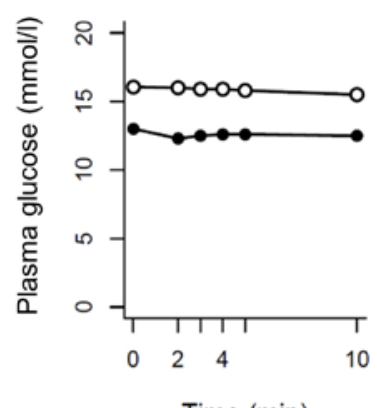

D

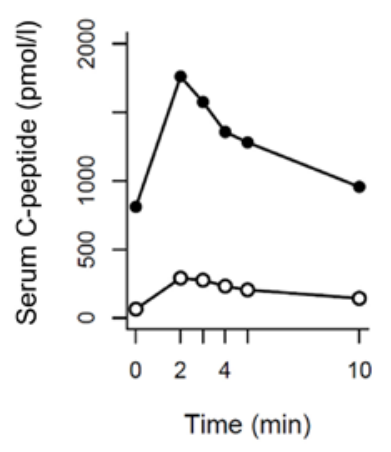

Figure 3. Plasma glucose and serum C-peptide concentrations during liquid mixed meal tests ( $A$ and $B$ ) and intravenous arginine stimulation tests (C and D), before initiation of glibenclamide treatment (white circles) and 9 months after initiation of glibenclamide treatment (black circles)

GLP-1 receptor agonists are only suitable as add-on therapy to sulfonylurea in the treatment of KCNJ11 neonatal diabetes.

This is because the insulinotropic effect of GLP-1 receptor agonists requires an already active insulin secretion pathway, which in the case of KCNJ11 mutations requires sulfonylurea treatment as described in the introduction and schematically illustrated in Fig. 1.

In conclusion, the present case emphasizes the importance of genetic testing in targeted treatment of permanent neonatal diabetes and introduces the possible use of GLP-1 receptor agonists as add-on therapy to sulfonylurea in patients with KCNJ11 mutations and inadequate effect of sulfonylurea monotherapy.

\section{REFERENCES}

1. Lemelman MB, Letourneau L, Greeley SAW. Neonatal diabetes mellitus: an update on diagnosis and management. Clin Perinatol 2018;45(1):41-59.

2. Proks P, Antcliff JF, Lippiat J, Gloyn AL, Hattersley AT, Ashcroft FM. Molecular basis of Kir6.2 mutations associated with neonatal diabetes or neonatal diabetes plus neurological features. Proc Natl Acad Sci U S A 2004;101(50):17539-17544.

3. Bowman P, Sulen Å, Barbetti F, Beltrand J, Svalastoga P, Codner E, et al. Effectiveness and safety of long-term treatment with sulfonylureas in patients with neonatal diabetes due to KCNJ11 mutations: an international cohort study. Lancet Diabetes Endocrinol 2018;6(8):637-646.

4. Gade-Rasmussen B, Madsbad S, Svane MS, Martinussen C, Hansen T. [Successful treatment of KCNJ11 neonatal diabetes without insulin]. Ugeskr Laeger 2018;180(46):V08180547.

5. Sastre J, Luque A, del Val F, Aragonés A, López J. Long-term efficacy of glibenclamide and sitagliptin therapy in adult patients with KCNJ11 permanent diabetes. Diabetes Care 2014;37(3):e55-56. 